\title{
Constraint qualifications in convex vector semi-infinite optimization*
}

\author{
M.A. Gobernał; F. Guerra-Vazquez $\ddagger$ and M.I. Todorov ${ }^{\S}$
}

February 17, 2015

\begin{abstract}
Convex vector (or multi-objective) semi-infinite optimization deals with the simultaneous minimization of finitely many convex scalar functions subject to infinitely many convex constraints. This paper provides characterizations of the weakly efficient, efficient and properly efficient points in terms of cones involving the data and Karush-Kuhn-Tucker conditions. The latter characterizations rely on different local and global constraint qualifications. The results in this paper generalize those obtained by the same authors on linear vector semi-infinite optimization problems [Eur J Oper Res 227 (2013) 12-21].
\end{abstract}

\section{Introduction}

We consider convex optimization problems of the form

$$
P: " \min " f(x)=\left(f_{1}(x), \ldots, f_{p}(x)\right) \text { s.t. } g_{t}(x) \leq 0, t \in T,
$$

where $x \in \mathbb{R}^{n}$ (the space of decisions), $f(x) \in \mathbb{R}^{p}$ (the objective space), the index set $T$ is a compact Hausdorf topological space, $f_{i}: \mathbb{R}^{n} \rightarrow \mathbb{R}$ is a convex function, $i=1, \ldots, p, g_{t}$ is convex for each $t \in T$, and the function $(t, x) \mapsto g_{t}(x)$ is continuous on $T \times \mathbb{R}^{n}$. The continuity of $f$ is consequence of the assumptions on its components $f_{1}, \ldots, f_{p}$. The model (1) includes ordinary convex (scalar and vector) optimization problems just taking the discrete topology on the (finite) index set. Since the optimality theory for this class of problems has been thoroughly studied, we assume in the sequel that $T$ is infinite. When $p \geq 2, P$ is

${ }^{*}$ This research was partially supported by MICINN of Spain, Grant MTM2011-29064-C0302, and Sistema Nacional de Investigadores, Mexico.

${ }^{\dagger}$ Dep. of Statistics and Operations Research, Alicante University, 03071 Alicante, Spain. E-Mail: mgoberna@ua.es.

${ }^{\ddagger}$ Dep. of Actuarial Sciences, Physics and Mathematics, UDLAP, 72820 San Andrés Cholula, Puebla, Mexico. E-Mail: francisco.guerra@udlap.mx.

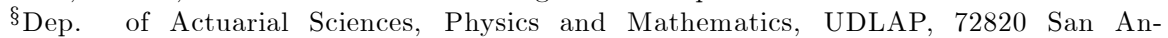
drés Cholula, Puebla, Mexico. On leave from IMI-BAS, Sofia, Bulgaria. E-Mail: maxim.todorov@udlap.mx. 
a convex vector semi-infinite optimization (SIO in brief) problem; otherwise, $P$ is a convex scalar SIO problem. Replacing in (1) the space of decisions $\mathbb{R}^{n}$ by an infinite dimensional space (typically a locally convex Hausdorff topological vector space) one gets a convex (scalar or vector) infinite optimization (IO in short) problem.

We assume throughout the paper that $p \geq 2$ and the feasible set of $P$, denoted by $X$, is non-empty. Obviously, $X$ is a closed convex set whereas its image by the vector-valued objective function $f(X) \subset \mathbb{R}^{p}$ is possibly non-convex and nonclosed. The vector SIO problem $P$ can be reformulated as a vector optimization problem with the single convex constraint function $\varphi(x):=\max _{t \in T} g_{t}(x)$, called marginal function:

$$
P: " \min " f(x)=\left(f_{1}(x), \ldots, f_{p}(x)\right) \text { s.t. } \varphi(x) \leq 0 .
$$

Throughout the paper we use the following notation. Given $x, y \in \mathbb{R}^{m}$, we write $x \leqq y(x<y)$ when $x_{i} \leq y_{i}\left(x_{i}<y_{i}\right.$, respectively) for all $i=1, \ldots, m$. Moreover, we write $x \leq y$ when $x \leqq y$ and $x \neq y$.

An element $\bar{x} \in X$ is said to be efficient (weakly efficient) if there is no $\widehat{x} \in X$ such that $f(\widehat{x}) \leq f(\bar{x})(f(\widehat{x})<f(\bar{x})$, respectively). There are many notions of proper efficiency in the literature, as those introduced by Geoffrion, Benson, Borwein and Henig. Since $P$ is convex, all these concepts are equivalent (see, e.g., [9]), so that we recall only Geoffrion's definition: a feasible point $\bar{x} \in X$ is said to be properly efficient if there exists $\rho>0$ such that, for all $i=1, \ldots, p$ and $\widehat{x} \in X$ satisfying $f_{i}(\widehat{x})<f_{i}(\bar{x})$, there exists $j \in\{1, \ldots, p\}$ such that $f_{j}(\widehat{x})>f_{j}(\bar{x})$ and $\frac{f_{i}(\bar{x})-f_{i}(\widehat{x})}{f_{j}(\widehat{x})-f_{j}(\bar{x})} \leq \rho$.

We denote by $X_{p E}, X_{E}$, and $X_{w E}$ the sets of properly efficient points, efficient points, and weakly efficient points of $P$, respectively. Obviously, $X_{p E} \subset$ $X_{E} \subset X_{w E}$, with $X=X_{w E}$ whenever one component of $f$ is identically zero, and $X=X_{p E}$ in the trivial case that $f$ is the null function. Moreover, it is known that $f\left(X_{p E}\right)$ is dense in $f\left(X_{E}\right)$ ([17]; see also [9, Theorem 3.17]).

Given a (possibly non-convex) vector SIO problem

$$
P: " \min " f(x) \text { s.t. } x \in X
$$

$\bar{x} \in X$ is said to be locally (properly, weakly) efficient solution of $P$ if there exists a neighborhood $\mathcal{N}$ of $\bar{x}$ such that $\bar{x}$ is (properly, weakly) efficient solution of

$$
P_{\mathcal{N}}: " \min " f(x) \text { s.t. } x \in X \cap \mathcal{N} \text {. }
$$

Global and local concepts coincide in convex vector SIO thanks to the convexity of $X$ and the componentwise convexity of $f$. For instance, if $\bar{x} \in X$ is not weakly efficient there exists $\widehat{x} \in X$ such that $f(\widehat{x})<f(\bar{x})$; since $f(\lambda \widehat{x}+(1-\lambda) \bar{x})<$ $f(\bar{x})$ for all $\lambda \in] 0,1[$, with $\lambda \widehat{x}+(1-\lambda) \bar{x} \in X \cap \mathcal{N}$ for $\lambda$ sufficiently small, $\bar{x}$ cannot be a locally weakly efficient solution of $P$. For this reason, in convex vector SIO, we can characterize the (proper, weak) efficiency on the basis of local information. The known tests for non-linear vector optimization classify 
a given $x \in X$ as locally (properly, weakly) efficient solution or not through conditions involving subsets of the objective space $\mathbb{R}^{p}$ or suitable scalarizations of $P$ (see, e.g., [2], [9]).

In this paper, on convex vector SIO, we give conditions for $\bar{x} \in X_{p E}, \bar{x} \in X_{E}$, and $\bar{x} \in X_{w E}$ which are expressed in terms of convex cones contained in the decision space $\mathbb{R}^{n}$ or in terms of the existence of Karush-Kuhn-Tucker (KKT in short) multipliers which can be computed from $\bar{x}$ and the data describing $P$.

As a general rule, to obtain a checkable necessary optimality condition for a given constrained optimization problem, one needs to assume some property of the constraint system called constraint qualification (CQ in short). We consider in this paper four CQs which extend those used in our previous paper [12] on linear vector SIO. The strongest one is the natural extension of the CQ introduced by M. Slater in a seminal work on scalar optimization published in 1950, which was adapted to linear scalar SIO by Charnes, Cooper and Kortanek in the 1960s. A weaker CQ for convex scalar SIO has been proposed in [23]. The locally Farkas-Minkowski CQ was first defined in [26] for linear scalar SIO, and then extended to convex scalar SIO in [13] and to convex scalar IO in [8]. CQs weaker than the locally Farkas-Minkowski one have been introduced in [23], for convex SIO problems, and in [22], for convex IO problems. The local Slater CQ, introduced in Section 3 of this paper, seems to be new while the extended Kuhn-Tucker CQ was introduced in [29] for convex IO as an extension of that used by H.W. Kuhn and A.W. Tucker in [19] for ordinary non-linear optimization problems. Section 1 of [22] reviews the state of the art on CQs in convex scalar optimization.

The stability of linear and non-linear vector SIO has been investigated since the last 1980s from different perspectives, e.g., the pseudo-Lipschitz property and the lower and upper semicontinuity of the efficient set mapping under different types of perturbations, well-posedness, and generic stability (see, e.g., [4], $[5],[6],[10],[30],[31])$, while the existing literature on optimality conditions for vector SIO and vector IO problems is surprisingly limited.

The main antecedent of this paper is [12], on linear vector SIO, which provides characterizations of the weakly efficient, efficient and properly efficient solutions in terms of cones involving the data and KKT conditions. In [3], on a class of vector SIO problems involving differentiable functions whose constraints satisfy certain invex-type conditions and are required to depend continuously on an index $t$ ranging on some compact topological space $T$, KKT conditions for $\bar{x} \in X_{p E}, \bar{x} \in X_{E}$ and $\bar{x} \in X_{w E}$ are given. In [14], on non-convex differentiable vector SIO, the authors discuss constraint qualifications as well as necessary and sufficient conditions for locally weakly efficient points and present optimality conditions for properly efficient points in the senses of Geoffrion and of Kuhn and Tucker [19]. Finally, in [7], on non-smooth vector IO problems posed on Asplund spaces whose index set $T$ has no topological structure, necessary conditions as well as sufficient conditions for weakly efficient solutions are obtained appealing to the machinery of non-smooth analysis and a certain CQ, for non-convex systems introduced in [4], which can be seen as an extension of the so-called basic CQs introduced in [21], for scalar IO problems posed in 


\section{Banach spaces.}

The convex vector SIO problems considered in this paper arise in a natural way in robust linear vector optimization. Indeed, consider an uncertain linear vector optimization problem

$$
(L P) \quad " \min "\left(c_{1}^{\top} x, \ldots, c_{p}^{\top} x\right) \text { s.t. } a_{t}^{\top} x \geq b_{t}, t \in T,
$$

where $T$ is a finite set, $c_{i} \in \mathcal{U}_{i} \subset \mathbb{R}^{n}, i=1, \ldots, p$, and $\left(a_{t}, b_{t}\right) \in \mathcal{V}_{t} \subset \mathbb{R}^{n+1}$, $t \in T$. The uncertainty sets $\mathcal{U}_{i}, i=1, \ldots, p$, are arbitrary non-empty sets while $\mathcal{V}_{t}, t \in T$, are non-empty compact sets. The robust minmax counterpart of $(L P)$ (term coined in [15]) enforces feasibility for any possible scenario and assumes that the cost of any (robust) feasible decision will be the worst possible, i.e., the problem to be solved is

$$
\text { " min " }\left(\max _{c_{1} \in \mathcal{U}_{1}} c_{1}^{\top} x, \ldots, \max _{c_{p} \in \mathcal{U}_{p}} c_{p}^{\top} x\right) \text { s.t. } a_{t}^{\top} x \geq b_{t}, \forall\left(a_{t}, b_{t}\right) \in \mathcal{V}_{t}, t \in T .
$$

Observe that (2) is as (1), just taking $f_{i}(x)=\max _{c_{i} \in \mathcal{U}_{i}} c_{i}^{\top} x$ (i.e., the support function of $\left.\mathcal{U}_{i}\right), i=1, \ldots, p$, and expressing the constraints either as $b-a^{\top} x \leq 0$ for all $(a, b) \in \bigcup_{t \in T} \mathcal{V}_{t}$ (a compact index set) or as $g_{t}(x) \leq 0$, with $g_{t}(x)=$ $\max \left\{b-a^{\top} x:(a, b) \in \bigcup_{t \in T} \mathcal{V}_{t}\right\}$ for all $t \in T$ (a finite index set equipped with the discrete topology).

This paper is organized as follows. Section 2 recalls basic concepts of convex analysis to be used later, applying some of them to characterize the subdifferential cone and its interior, and to describe the relationships between several types of "tangent" cones which are closely related with the negative polar of the active cone. Section 3 extends to convex vector SIO four out of six constraint qualifications introduced in [12] for linear vector SIO. The two exceptions, the Farkas-Minkowski and the local polyhedral constraint qualifications, have not been considered in this paper as they are too strong in the convex framework. For methodological reasons, we give simple direct proofs of the lemmas in Section 3 even though most of them could be also obtained via linearization. Finally, Section 4 provides different characterizations of the sets $X_{p E}, X_{E}$, and $X_{w E}$ in terms of cones and KKT multipliers. Here the proofs are necessarily direct as the objective functions are not linear. These results are applied to the robust linear vector optimization problem $(L P)$.

\section{Preliminaries}

We start this section by introducing the necessary notation and concepts. Given $Z \subset \mathbb{R}^{n}$, int $Z, \operatorname{cl} Z$, and bd $Z$ denote the interior, the closure, and the boundary of $Z$, respectively. The scalar product of $x, y \in \mathbb{R}^{n}$ is denoted by $x^{\top} y$, the Euclidean norm of $x$ by $\|x\|$, the corresponding open ball centered at $x$ and radius $\varepsilon>0$ by $B(x, \varepsilon)$, and the zero vector by $0_{n}$. We also denote by $\operatorname{conv} Z$ 
the convex hull of $Z$, while cone $Z:=\mathbb{R}_{+}$conv $Z$ denotes the convex conical hull of $Z \cup\left\{0_{n}\right\}$. If $Z$ is a convex cone, its positive (negative) polar cone is $Z^{+}:=$ $\left\{d \in \mathbb{R}^{n}: z^{\top} d \geq 0 \forall z \in Z\right\}\left(Z^{-}:=\left\{d \in \mathbb{R}^{n}: z^{\top} d \leq 0 \forall z \in Z\right\}\right.$, respectively). A convex cone is said to be pointed whenever it does not contain lines. We use frequently in this paper the topological interior of polar cones.

We make three claims concerning cone $Z$, where $Z$ is an arbitrary non-empty set of $\mathbb{R}^{n}$ :

First,

$$
0_{n} \notin \operatorname{conv} Z \Leftrightarrow\left\{\operatorname{cone} Z \text { is pointed and } 0_{n} \notin Z\right\} \text {. }
$$

We shall prove that $0_{n} \in$ conv $Z$ if and only if cone $Z$ contains lines or $0_{n} \in Z$.

If cone $Z$ contains lines, there exists $u \in \mathbb{R}^{n} \backslash\left\{0_{n}\right\}$ such that $\pm u \in$ cone $Z$. Then, we can write $u=\sum_{i=1}^{n} \alpha_{i} z_{i}$ and $-u=\sum_{i=1}^{n} \beta_{i} z_{i}$, with $\alpha_{1}, \ldots, \alpha_{n}, \beta_{1}, \ldots, \beta_{n} \in$ $\mathbb{R}_{+}, z_{i} \in Z, i=1, \ldots, n$, so that

$$
0_{n}=\left(\sum_{i=1}^{n}\left(\alpha_{i}+\beta_{i}\right)\right)^{-1} \sum_{i=1}^{n}\left(\alpha_{i}+\beta_{i}\right) z_{i} \in \operatorname{conv} Z .
$$

Alternatively, if $0_{n} \in Z$, it is obvious that $0_{n} \in \operatorname{conv} Z$.

Conversely, if $0_{n} \in \operatorname{conv} Z$, there exist $\alpha_{i} \in \mathbb{R}_{+}$and $z_{i} \in Z, i=1, \ldots, n$, such that $\sum_{i=1}^{n} \alpha_{i}=1$ and $\sum_{i=1}^{n} \alpha_{i} z_{i}=0_{n}$. Let $j \in\{1, \ldots, n\}$ be such that $\alpha_{j}>0$. If $z_{j} \neq 0_{n}$, putting $\alpha:=\sum_{i \neq j} \alpha_{i}>0$, it follows that $u:=-\frac{\alpha_{j}}{\alpha} z_{j}=\sum_{i \neq j} \frac{\alpha_{i}}{\alpha} z_{i} \in$ cone $Z$, so that cone $Z$ contains the line spanned by $u$. Now, suppose that $z_{j}=0_{n}$. Then

$$
\sum_{i \neq j} \alpha_{i} z_{i}=0_{n}
$$

If $\alpha_{i}=0$ for all $i \neq j$, then $0_{n}=z_{j} \in Z$. Otherwise, there exists $k \neq j$ such that $\alpha_{k}>0$, in (4), and we repeat the argument above.

Second,

$$
0_{n} \notin Z \Rightarrow \operatorname{int}(\text { cone } Z)^{+} \subset\left\{d \in \mathbb{R}^{n}: z^{\top} d>0 \forall z \in Z\right\} .
$$

In fact, assume that $0_{n} \notin Z$ and $d \in \operatorname{int}(\operatorname{cone} Z)^{+}$. Let $\varepsilon>0$ be such that $\operatorname{cl} B(d, \varepsilon) \subset(\text { cone } Z)^{+}$. Given $z \in Z, d-\varepsilon \frac{z}{\|z\|} \in(\text { cone } Z)^{+}$while $z \in$ cone $Z$, so that $z^{\top}\left(d-\varepsilon \frac{z}{\|z\|}\right)=z^{\top} d-\varepsilon\|z\| \geq 0$ and so $z^{\top} d \geq \varepsilon\|z\|>0$. Obviously, if $Z \cap(-Z) \neq \emptyset$, then both members of the inclusion in (5) are empty.

Third,

$$
Z \text { compact } \Rightarrow\left\{d \in \mathbb{R}^{n}: z^{\top} d>0 \forall z \in Z\right\} \subset \operatorname{int}(\operatorname{cone} Z)^{+} .
$$

In fact, by assumption, there exists $\delta>0$ such that $\|z\| \leq \delta$ for all $z \in Z$. Let $d \in \mathbb{R}^{n}$ be such that $z^{\top} d>0$ for all $z \in Z$. By the compactness of $Z$, 
$\varepsilon:=\min _{z \in Z} z^{\top} d>0$. Given $x \in$ cone $Z$, we can write $x=\sum_{i=1}^{n} \mu_{i} z_{i}$, with $\mu_{i} \geq 0$ and $z_{i} \in Z, i=1, \ldots, n$. Then, given $u$ such that $\|u\| \leq 1$, one has

$$
x^{\top}\left(d+\frac{\varepsilon}{\delta} u\right)=\sum_{i=1}^{n} \mu_{i} z_{i}^{\top}\left(d+\frac{\varepsilon}{\delta} u\right) \geq \sum_{i=1}^{n} \mu_{i}\left(\varepsilon-\frac{\varepsilon}{\delta}\left|z^{\top} u\right|\right) \geq 0 .
$$

Thus, $B\left(d, \frac{\varepsilon}{\delta}\right) \subset(\text { cone } Z)^{+}$and $d \in \operatorname{int}(\text { cone } Z)^{+}$. The inclusion in (6) becomes an equation between non-empty sets whenever $Z$ is compact and $0_{n} \notin \operatorname{conv} Z$ (as cone $Z$ turns out to be a pointed cone).

The one-sided directional derivative of a real-valued function $h: \mathbb{R}^{n} \rightarrow \mathbb{R}$ at $\bar{x} \in \mathbb{R}^{n}$ with respect to a vector $d \in \mathbb{R}^{n}$ is defined to be the limit

$$
h^{\prime}(\bar{x} ; d)=\lim _{\varepsilon \downarrow 0} \frac{h(\bar{x}+\varepsilon d)-h(\bar{x})}{\varepsilon},
$$

if it exists. If $h$ is convex, then it is continuous, the directional derivative function at $\bar{x} \in \mathbb{R}^{n}, h^{\prime}(\bar{x} ;$.$) , is a finite convex function too, and the subdifferential$

$$
\partial h(\bar{x}):=\left\{\xi \in \mathbb{R}^{n}: h(x) \geq h(\bar{x})+\xi^{\top}(x-\bar{x}) \forall x \in \mathbb{R}^{n}\right\},
$$

is a non-empty compact convex such that $h^{\prime}(\bar{x} ; d)=\max _{\xi \in \partial h(\bar{x})} \xi^{\top} d$ (see, e.g., [27, Theorems 23.1 and 23.4]).

Lemma 1 Let $h: \mathbb{R}^{n} \rightarrow \mathbb{R}$ be a convex function and let $d \in \mathbb{R}^{n}$ be a descent direction of $h$ at $\bar{x} \in \mathbb{R}^{n}$. Then, $h^{\prime}(\bar{x} ; d)<0$.

Proof: Since $d$ is a descent direction of $h$ at $\bar{x}$ there exists $\beta>0$ such that

$$
h(\bar{x}+\varepsilon d)-h(\bar{x})<0 \text { for all } \varepsilon \in] 0, \beta[.
$$

Thus

$$
h^{\prime}(\bar{x} ; d)=\lim _{\varepsilon \downarrow 0} \frac{h(\bar{x}+\varepsilon d)-h(\bar{x})}{\varepsilon} \leq 0 .
$$

On the other hand

$$
h^{\prime}(\bar{x} ; d)=\max _{\xi \in \partial h(\bar{x})} \xi^{\top} d .
$$

Now, taking into account that $0_{n} \notin \partial h(\bar{x})$ and $\partial h(\bar{x})$ is compact, if $h^{\prime}(\bar{x} ; d)=0$ there would exists $\widetilde{\xi} \in \partial h(\bar{x}), \widetilde{\xi} \neq 0_{n}$ such that $\widetilde{\xi}^{\top} d=0$. Thus, in particular, for $\widetilde{\xi}$ and $\bar{x}+\varepsilon d$ with $\varepsilon \in] 0, \beta[$

$$
h(\bar{x}+\varepsilon d)-h(\bar{x}) \geq \varepsilon \widetilde{\xi}^{\top} d=0
$$

which contradicts (7).

It is easy to prove that, under the assumptions on $P$,

$$
X=\left\{x \in \mathbb{R}^{n}: \xi^{\top} x \leq \xi^{\top} y-g_{t}(y), \forall(t, y) \in T \times \mathbb{R}^{n}, \forall \xi \in \partial g_{t}(y)\right\} .
$$


From (8) and [13, Theorem 9.3] one gets that $X$ is compact if and only if cone $\left(\bigcup_{(t, y) \in T \times \mathbb{R}^{n}} \partial g_{t}(y)\right)=\mathbb{R}^{n}$. This condition (expressed in terms of the data), guarantees the compactness of $f(X)$. More information on $X$ can be obtained from the linearization (8) of $X$ under the constraint qualifications introduced in Section 3 .

Two convex cones involving the data of the vector SIO problem $P$ in (1) are basic in our approach: the convex conical hull of the subdifferentials at $\bar{x} \in X$ of the components of $f$,

$$
G(\bar{x}):=\text { cone }\left(\bigcup_{i=1}^{p} \partial f_{i}(\bar{x})\right),
$$

that we shall call subdifferential cone at $\bar{x}$, and the active cone at $\bar{x} \in X$,

$$
A(\bar{x}):=\text { cone }\left(\bigcup_{t \in T(\bar{x})} \partial g_{t}(\bar{x})\right),
$$

where $T(\bar{x}):=\left\{t \in T: g_{t}(\bar{x})=0\right\}$ is the set of active indices at $\bar{x}$.

We are interested in the negative polar of both cones, $G(\bar{x})^{-}$and $A(\bar{x})^{-}$, and their corresponding interiors, int $G(\bar{x})^{-}$and int $A(\bar{x})^{-}$.

Lemma 2 Given $\bar{x} \in X$, the following statements hold:

(i) $0_{n} \notin \operatorname{conv}\left(\bigcup_{i=1}^{p} \partial f_{i}(\bar{x})\right)$ if and only if $G(\bar{x})$ is pointed and $0_{n} \notin \bigcup_{i=1}^{p} \partial f_{i}(\bar{x})$.

(ii) $G(\bar{x})^{-}=\left\{d \in \mathbb{R}^{n}: f_{i}^{\prime}(\bar{x} ; d) \leq 0, i=1, \ldots, p\right\}$.

(iii) If $0_{n} \notin \bigcup_{i=1}^{p} \partial f_{i}(\bar{x})$, then

$$
\begin{aligned}
\operatorname{int} G(\bar{x})^{-} & =\left\{d \in \mathbb{R}^{n}: \xi^{\top} d<0 \forall \xi \in \bigcup_{i=1}^{p} \partial f_{i}(\bar{x})\right\} \\
& =\left\{d \in \mathbb{R}^{n}: f_{i}^{\prime}(\bar{x} ; d)<0, i=1, \ldots, p\right\},
\end{aligned}
$$

with $\operatorname{int} G(\bar{x})^{-} \neq \emptyset$ whenever $0_{n} \notin \operatorname{conv}\left(\bigcup_{i=1}^{p} \partial f_{i}(\bar{x})\right)$.

Proof: (i) It is straightforward consequence of (3), just taking $Z=\bigcup_{i=1}^{p} \partial f_{i}(\bar{x})$.

(ii) Since $f_{i}^{\prime}(\bar{x} ; d)=\max _{\xi \in \partial f_{i}(\bar{x})} \xi^{\top} d$, one has that $\xi^{\top} d \leq 0$ for all $\xi \in \partial f_{i}(\bar{x})$ if and only if $f_{i}^{\prime}(\bar{x} ; d) \leq 0, i=1, \ldots, p$. So,

$$
\begin{aligned}
G(\bar{x})^{-} & =\left\{d \in \mathbb{R}^{n}: \xi^{\top} d \leq 0 \forall \xi \in \bigcup_{i=1}^{p} \partial f_{i}(\bar{x})\right\} \\
& =\left\{d \in \mathbb{R}^{n}: f_{i}^{\prime}(\bar{x} ; d) \leq 0, i=1, \ldots, p\right\},
\end{aligned}
$$


(iii) Again by the identity $f_{i}^{\prime}(\bar{x} ; d)=\max _{\xi \in \partial f_{i}(\bar{x})} \xi^{\top} d$, one has that $\xi^{\top} d<0$ for all $\xi \in \partial f_{i}(\bar{x})$ if and only if $f_{i}^{\prime}(\bar{x} ; d)<0, i=1, \ldots, p$. Assuming that $0_{n} \notin \bigcup_{i=1}^{p} \partial f_{i}(\bar{x})$, from $(5)$ and $(6)$, one gets $(9)$. The additional condition that $0_{n} \notin \operatorname{conv}\left(\bigcup_{i=1}^{p} \partial f_{i}(\bar{x})\right)$ guarantees, by (i), that $G(\bar{x})$ is pointed, which in turn implies that int $G(\bar{x})^{-} \neq \emptyset$.

The KKT conditions will be obtained by analyzing the relationships between negative polar of the active cone $A(\bar{x})$ and four "tangent" cones at $\bar{x}$ defined as follows:

The cone of feasible directions at $\bar{x}$ is

$$
D(X ; \bar{x})=\left\{d \in \mathbb{R}^{n}: \exists \mu>0 \text { such that } \bar{x}+\mu d \in X\right\} .
$$

It is known that $D(X ; \bar{x}) \subset A(\bar{x})^{-}([13$, Lemma 7.7]).

The attainable cone at $\bar{x}$, denoted by $A(X ; \bar{x})$, is formed by those $d \in \mathbb{R}^{n}$ such that there exist $\tau>0$ and a vector function $h \in \mathcal{C}^{1}\left(\left[0, \tau\left[, \mathbb{R}^{n}\right)\right.\right.$ with $h(0)=\bar{x}$, $h^{\prime}(0)=d$, and $h(s) \in X$ for all $s \in[0, \tau[$.

The Bouligand tangent cone at $\bar{x}$, denoted by $T(X ; \bar{x})$, is formed by those $d \in \mathbb{R}^{n}$ such that there exist sequences $\left\{s^{k}\right\}_{k \in \mathbb{N}}$ and $\left\{d^{k}\right\}_{k \in \mathbb{N}}$ such that $s^{k} \downarrow 0$, $d^{k} \rightarrow d$ as $k \rightarrow \infty$ and $\bar{x}+s^{k} d^{k} \in X$ for all $k \in \mathbb{N}$. In that case, since $d^{k} \in D(X ; \bar{x})$ for all $k \in \mathbb{N}, d \in \operatorname{cl} D(X ; \bar{x})$.

The interior tangent cone at $\bar{x}$, denoted by $T^{i}(X ; \bar{x})$, is formed by those $d \in \mathbb{R}^{n}$ such that there exist $\tau>0$ and a neighborhood $\mathcal{N}$ of $d$ such that $\bar{x}+s \mathcal{N} \subset X$ for all $s \in] 0, \tau[$.

Lemma 3 ([2], [16], [20], [25], [27], [32]) Given $\bar{x} \in X$, the cones $T^{i}(Z ; x)$, $D(Z ; x), A(Z ; x)$, and $T(Z ; x)$ are all convex and satisfy

$$
T^{i}(X ; \bar{x})=\operatorname{int} D(X ; \bar{x}) \subset D(X ; \bar{x}) \subset A(X ; \bar{x})=T(X ; \bar{x})=\operatorname{cl} D(X ; \bar{x}) .
$$

Example 4 Consider the closed convex set

$$
X=\left\{\left(x_{1}, x_{2}\right) \in \mathbb{R}^{2}: t\left(x_{1}-2\right)^{2}-t-x_{2} \leq 0 \forall t \in[0,1]\right\}
$$

and the point $\bar{x}=(1,0) \in X$. Since

$$
\varphi(x)= \begin{cases}-x_{2}, & 1 \leq x_{1} \leq 3, \\ x_{1}^{2}-4 x_{1}+3-x_{2}, & \text { otherwise }\end{cases}
$$

one has

$$
X=\left\{x \in \mathbb{R}^{2}: \varphi(x) \leq 0\right\}=\left\{x \in \mathbb{R}^{2}: x_{2} \geq \max \left\{x_{1}^{2}-4 x_{1}+3,0\right\}\right\},
$$

so that

$$
\begin{gathered}
D(X ; \bar{x})=\left\{d \in \mathbb{R}^{2}: 2 d_{1}+d_{2}>0, d_{2} \geq 0\right\} \cup\left\{0_{2}\right\}, \\
A(X ; \bar{x})=T(X ; \bar{x})=\left\{d \in \mathbb{R}^{2}: 2 d_{1}+d_{2} \geq 0, d_{2} \geq 0\right\}=\operatorname{cl} D(X ; \bar{x}),
\end{gathered}
$$


and

$$
T^{i}(X ; \bar{x})=\left\{d \in \mathbb{R}^{2}: 2 d_{1}+d_{2}>0, d_{2}>0\right\}=\operatorname{int} D(X ; \bar{x}) .
$$

Hence, the inclusions in (11) are strict. Observe that

$$
A(\bar{x})=\text { cone }\{(-2 t,-1): t \in[0,1]\}=\text { cone }\{(0,-1),(-2,-1)\}
$$

is the negative polar of any of the cones considered in Lemma 3.

Lemma 5 Given $\bar{x} \in X$,

$$
A(\bar{x})^{-}=\left\{d \in \mathbb{R}^{n}: \varphi^{\prime}(\bar{x} ; d) \leq 0\right\}
$$

and

$$
\operatorname{int} A(\bar{x})^{-}=\left\{d \in \mathbb{R}^{n}: \varphi^{\prime}(\bar{x} ; d)<0\right\}
$$

Proof: One has

$$
\begin{aligned}
\varphi^{\prime}(\bar{x} ; d) & =\max _{t \in T(\bar{x})} \max _{\xi \in \partial g_{t}(\bar{x})} \xi^{\top} d \\
& =\max \left\{\xi^{\top} d: \xi \in \bigcup_{t \in T(\bar{x})} \partial g_{t}(\bar{x})\right\} .
\end{aligned}
$$

From (12), we have $\varphi^{\prime}(\bar{x} ; d) \leq 0$ if and only if $\xi^{\top} d \leq 0$ for all $\xi \in \bigcup_{t \in T(\bar{x})} \partial g_{t}(\bar{x})$ if and only if $d \in A(\bar{x})^{-}$.

Similarly, by the compactness of $\bigcup_{t \in T(\bar{x})} \partial g_{t}(\bar{x}), \varphi^{\prime}(\bar{x} ; d)<0$ if and only if $\xi^{\top} d<0$ for all $\xi \in \bigcup_{t \in T(\bar{x})} \partial g_{t}(\bar{x})$ if and only if $d \in \operatorname{int} A(\bar{x})^{-}$.

\section{Constraint qualifications}

Next we introduce four constraint qualifications which are frequently encountered in the SIO literature or are inspired in classical constraint qualifications of non-linear optimization. When the constraint functions are affine, these constraint qualifications collapse to those introduced, under similar names, in [12] (for linear vector SIO). Even more, the below CQ hold for the convex system $\left\{g_{t}(x) \leq 0, t \in T\right\}$ if and only if the corresponding linear versions hold for the linear system in (8).

Definition 6 We say that P satisfies the Slater constraint qualification (SCQ) if there is a Slater point $x^{0}$, i.e. $g_{t}\left(x^{0}\right)<0$, for all $t \in T$.

In other words, SCQ holds if and only if the marginal function $\varphi$ takes a negative value at some point (observe that the marginal function of the linear system in (8) is also $\varphi$ ). By continuity of $\varphi$, SCQ implies that int $X \neq \emptyset$, but the converse is not true. It is worth noting that, in contrast with the other three CQs to be introduced next, SCQ is not associated with a given feasible solution. 
Definition 7 We say that the locally Farkas-Minkowski constraint qualification (LFMCQ) holds at $\bar{x} \in X$ if $A(\bar{x})=D(X ; \bar{x})^{-}$.

Obviously, if LFMCQ holds at $\bar{x} \in X$, then $A(\bar{x})$ is closed. If $\bar{x} \in \operatorname{int} X$, then $A(\bar{x})=D(X ; \bar{x})^{-}=\left\{0_{n}\right\}$. Therefore the LFMCQ should be investigated only at the boundary feasible points. Moreover, if this property holds and $\bar{x} \in$ bd $X$, we have $A(\bar{x}) \neq\left\{0_{n}\right\}$, i.e. there are binding constraints at $\bar{x}$.

Lemma 8 ([13, Theorem 7.9]) SCQ implies LFMCQ at any feasible solution.

Consequently, if $P$ satisfies SCQ, then bd $X=\left\{x \in X: A(x) \neq\left\{0_{n}\right\}\right\}$. Moreover, geometric information on $X$ in terms of the data can be obtained by combining (8) and [13, Theorem 5.9].

Definition 9 We say that $P$ satisfies the local Slater constraint qualification (LSCQ) at $\bar{x} \in X$ when either $T(\bar{x})=\emptyset$ or there exists a vector $d \in \mathbb{R}^{n}$ satisfying

$$
d^{\top} \xi<0 \text { for all } \xi \in \bigcup_{t \in T(\bar{x})} \partial g_{t}(\bar{x}) .
$$

Proposition 10 LSCQ holds at $\bar{x} \in X$ if and only if

$$
T(\bar{x}) \neq \emptyset \Rightarrow 0_{n} \notin \operatorname{conv}\left(\bigcup_{t \in T(\bar{x})} \partial g_{t}(\bar{x})\right) .
$$

Proof: We can assume that $T(\bar{x}) \neq \emptyset$. The direct statement is obvious while the converse statement is consequence of the assumptions on $P$. In fact, the continuity of $t \mapsto g_{t}(\bar{x})$ on the compact set $T$ entails that $T(\bar{x})$ is a compact set, as well as $\bigcup_{t \in T(\bar{x})} \partial g_{t}(\bar{x})$ (see e.g. [18, Theorem 4.4.2]). Due to the compactness of conv $\left(\bigcup_{t \in T(\bar{x})} \partial g_{t}(\bar{x})\right)$ and the separation theorem, the condition $0_{n} \notin \operatorname{conv}\left(\bigcup_{t \in T(\bar{x})} \partial g_{t}(\bar{x})\right)$ guarantees the fulfilment of LSCQ at $\bar{x}$.

Corollary 11 If $P$ satisfies $L S C Q$ at $\bar{x} \in X$, then $A(\bar{x})$ is a pointed closed cone.

Proof: We can assume $T(\bar{x}) \neq \emptyset$ (otherwise $A(\bar{x})=\left\{0_{n}\right\}$ is closed). By Proposition 10, since conv $\left(\bigcup_{t \in T(\bar{x})} \partial g_{t}(\bar{x})\right)$ is a compact convex set which does 
not contain the origin, $A(\bar{x})=$ cone $\left(\bigcup_{t \in T(\bar{x})} \partial g_{t}(\bar{x})\right)$ is a pointed closed cone.

The following example shows that the converse statement of Corollary 11 does not hold.

Example 12 Let $n=2$ and $g_{t}(x)=(1-t)\left|x_{1}-1\right|+\left|x_{2}\right|-1+t$ for all $T=[0,1]$. Then it is easy to see that

$$
X=\left\{x \in \mathbb{R}^{2}: g_{t}(x) \leq 0, t=0,1\right\}=[0,2] \times\{0\} .
$$

We have $T\left(0_{2}\right)=[0,1]$, with

$$
\partial g_{t}\left(0_{2}\right)=\operatorname{conv}\{(t-1,-1),(t-1,1)\}=\{t-1\} \times[-1,1], t \in[0,1] .
$$

Thus, $\bigcup_{t \in T\left(0_{2}\right)} \partial g_{t}\left(0_{2}\right)=[-1,0] \times[-1,1]$ and $A\left(0_{2}\right)=$ cone $\left(\bigcup_{t \in T\left(0_{2}\right)} \partial g_{t}\left(0_{2}\right)\right)=$ $\mathbb{R}_{-} \times \mathbb{R}$ is closed. Finally, as $\pm(0,1) \in \partial g_{0}\left(0_{2}\right)$, LSCQ fails at $0_{2}$.

Definition 13 We say that $P$ satisfies the extended Kuhn-Tucker CQ (EK$T C Q$ ) at $\bar{x} \in X$ when

$$
\left\{d \in \mathbb{R}^{n}: \varphi^{\prime}(\bar{x} ; d) \leq 0\right\} \subset A(X ; \bar{x}) .
$$

As a consequence of the Ioffe and Tihkomirov's theorem on the subdifferential of the supremum function (see e.g. [32, Theorem 2.4.18] and [16, Proposition 6.3]), when $\bar{x} \in$ bd $X$, it holds that

$$
\varphi^{\prime}(\bar{x} ; d)=\max _{t \in T(\bar{x})} g_{t}^{\prime}(\bar{x} ; d) .
$$

The next lemma provides a useful approximations of the tangent cones to $X$ at $\bar{x} \in \operatorname{bd} X$ in terms of the directional derivative function $\varphi^{\prime}(\bar{x} ; \cdot)$.

Lemma 14 Let $\bar{x} \in \operatorname{bd} X$. Then,

$$
\left\{d \in \mathbb{R}^{n}: \varphi^{\prime}(\bar{x}, d)<0\right\} \subset T^{i}(X ; \bar{x}) \subset T(X ; \bar{x}) \subset\left\{d \in \mathbb{R}^{n}: \varphi^{\prime}(\bar{x}, d) \leq 0\right\} .
$$

Proof: We first show that $\left\{d \in \mathbb{R}^{n}: \varphi^{\prime}(\bar{x}, d)<0\right\} \subset T^{i}(X ; \bar{x})$. Let $\bar{d} \in \mathbb{R}^{n}$ be such that $\varphi^{\prime}(\bar{x}, \bar{d})<0$. Then, there exists $\tau>0$ such that $\varphi(\bar{x}+s \bar{d})<0$ for all $s \in] 0, \tau\left[\right.$. By continuity of $\varphi$, there exist $t_{0}>0$ and an open neighborhood $\mathcal{N}$ of $\bar{d}$ such that $\varphi(\bar{x}+t d)<0$ for all $t \in] 0, t_{0}[$ and all $d \in \mathcal{N}$. That is,

$$
\bar{d} \in T^{i}\left(\left\{x \in \mathbb{R}^{n}: \varphi(x)<0\right\}, \bar{x}\right) \subset T^{i}\left(\left\{x \in \mathbb{R}^{n}: \varphi(x) \leq 0\right\}, \bar{x}\right)=T^{i}(X ; \bar{x}) .
$$

The inclusion $T^{i}(X ; \bar{x}) \subset T(X ; \bar{x})$ is well-known (see, e.g. in [28]). 
Finally, consider $\bar{d} \in T(X ; \bar{x})$. Then, there exist sequences $\left\{s^{k}\right\}_{k \in \mathbb{N}}$ and $\left\{d^{k}\right\}_{k \in \mathbb{N}}$ such that $s^{k} \downarrow 0, d^{k} \rightarrow \bar{d}$ as $k \rightarrow \infty$ and $\varphi\left(\bar{x}+s^{k} d^{k}\right) \leq 0$ for all $k \in \mathbb{N}$. Since $\varphi(\bar{x})=0$, one has

$$
\frac{\varphi\left(\bar{x}+s^{k} d^{k}\right)}{s^{k}}=\frac{\varphi\left(\bar{x}+s^{k} d^{k}\right)-\varphi(\bar{x})}{s^{k}} \leq 0 \forall k \in \mathbb{N} .
$$

Now, taking limits as $k \rightarrow \infty$, we conclude that

$$
\frac{\varphi\left(\bar{x}+s^{k} d^{k}\right)}{s^{k}} \rightarrow \varphi^{\prime}(\bar{x} ; \bar{d}) \leq 0 .
$$

Theorem 15 The following statements are true:

(i) $S C Q$ implies $L S C Q$ at any $\bar{x} \in X$.

(ii) If $L S C Q$ holds at $\bar{x} \in X$ and $T(\bar{x})$ is a set of isolated points of $T$, then $S C Q$ holds.

(iii) If LSCQ holds at $\bar{x} \in X$, then $L F M C Q$ holds at $\bar{x}$.

(iv) If LSCQ holds at $\bar{x} \in X$, then EKTCQ holds at $\bar{x}$.

Proof: (i) Let $x^{0}$ be a Slater point and $\bar{x} \in X$. Let $d:=x^{0}-\bar{x}$ and $\xi \in \partial g_{t}(\bar{x})$ for some $t \in T(\bar{x})$. Since

$$
0>g_{t}\left(x^{0}\right) \geq g_{t}(\bar{x})+\xi^{\top}\left(x^{0}-\bar{x}\right)=d^{\top} \xi,
$$

$d$ satisfies (13).

(ii) We shall prove that, under the assumption (equivalent to assert that $T(\bar{x})$ is finite and $T \backslash T(\bar{x})$ is compact), there exists a Slater point in the half-line emanating from $\bar{x}$ in some direction $d$ satisfying (13). In fact, given $t \in T(\bar{x})$, as $g_{t}^{\prime}(\bar{x} ; d)=\max _{\xi \in \partial g_{t}(\bar{x})} \xi^{\top} d<0$, there exists $\varepsilon_{t}>0$ with $g_{t}(\bar{x}+\lambda d)<0$ for any $\lambda \in] 0, \varepsilon_{t}\left[\right.$. On the other hand, by continuity of the function $\max _{t \in T \backslash T(\bar{x})} g_{t}$, there exists a neighborhood $\mathcal{N}$ of $\bar{x}$ where $\max _{t \in T \backslash T(\bar{x})} g_{t}$ is negative. Taking a sufficiently small $\lambda_{0}>0$, we get $g_{t}\left(\bar{x}+\lambda_{0} d\right)<0$ for all $t \in T(\bar{x})$ and $\bar{x}+\lambda_{0} d \in$ $\mathcal{N}$. So, $\bar{x}+\lambda_{0} d$ is a Slater point.

(iii) By a well-known result (see, e.g., [24, Proposition 5]), if $\varphi^{\prime}(\bar{x} ; d)<0$, then $d \in D(X ; \bar{x})$. This, combined with Lemma 5 , yields

$$
\operatorname{int} A(\bar{x})^{-} \subset D(X ; \bar{x}),
$$

where int $A(\bar{x})^{-} \neq \emptyset$ by Corollary 11, as the negative polar of a pointed closed convex cone contains interior points. Taking negative polars in both members of (16) one gets, by the Farkas lemma for cones,

$$
D(X ; \bar{x})^{-} \subset\left(\operatorname{int} A(\bar{x})^{-}\right)^{-}=\left(A(\bar{x})^{-}\right)^{-}=\operatorname{cl} A(\bar{x})=A(\bar{x}) .
$$

We now prove the reverse inclusion by contradiction. Suppose that there exists $\xi \in A(\bar{x}) \backslash D(X ; \bar{x})^{-}$. Then there exists $d \in D(X ; \bar{x})$ such that $\xi^{\top} d>0$, 
with $\xi=\sum_{i=1}^{m} \xi_{i}, \xi_{i} \in \partial g_{t_{i}}(\bar{x}), t_{i} \in T(\bar{x}), i=1, \ldots, m$. Let $i_{0} \in\{1, \ldots, m\}$ such that $\xi_{i_{0}}^{\top} d>0$. This means that for any $\varepsilon>0$, we have

$$
g_{t_{i_{0}}}(\bar{x}+\varepsilon d)=g_{t_{i_{0}}}(\bar{x}+\varepsilon d)-g_{t_{i_{0}}}(\bar{x}) \geq \varepsilon \xi_{i_{0}}^{\top} d>0,
$$

so that $d \notin D(X ; \bar{x})$ (contradiction). Thus, $A(\bar{x})=D(X ; \bar{x})^{-}$.

(iv) Let $\bar{x} \in \operatorname{int} X$. If $\bar{x} \in \operatorname{int} X$, then $A(X ; \bar{x})=\mathbb{R}^{n}$ and (14) holds trivially. Thus we can assume without loss of generality (w.l.o.g. in short) that $\bar{x} \in \mathrm{bd} X$.

Let $d \in \mathbb{R}^{n}$ satisfy (13). By (15), we have, from (12),

$$
\varphi^{\prime}(\bar{x} ; d)=\max \left\{\xi^{\top} d: \xi \in \bigcup_{t \in T(\bar{x})} \partial g_{t}(\bar{x})\right\}<0 .
$$

Since $\left\{d \in \mathbb{R}^{n}: \varphi^{\prime}(\bar{x} ; d)<0\right\} \neq \emptyset$ and $\varphi^{\prime}(\bar{x} ; \cdot)$ is a finite-valued convex function ([27, Theorem 23.4]), we get

$$
\operatorname{cl}\left\{d \in \mathbb{R}^{n}: \varphi^{\prime}(\bar{x} ; d)<0\right\}=\left\{d \in \mathbb{R}^{n}: \varphi^{\prime}(\bar{x} ; d) \leq 0\right\} .
$$

Then, by Lemma 14 and Lemma 3,

$$
\left\{d \in \mathbb{R}^{n}: \varphi^{\prime}(\bar{x} ; d) \leq 0\right\}=T(X ; \bar{x})=A(X ; \bar{x}),
$$

and so EKTCQ holds at $\bar{x}$.

Observe that the assumptions on $T(\bar{x})$ and $T \backslash T(\bar{x})$ in Theorem 15(ii) are not superfluous (see [12, Example 4]) and imply the non-connectedness of $T$. In the particular case that $T$ is finite, SCQ and LSCQ are equivalent. Notice also that Lemma 8 follows straighforwardly from statements (i) and (iii) of Theorem 15.

The next example shows that LFMCQ does not imply LSCQ (consequently does not imply SCQ).

Example 16 Let $n=2$ and $g_{t}(x)=\|x\|-t$ for all $t \in T=[0,1]$. It follows that $X=\left\{0_{2}\right\}$ and $T\left(0_{2}\right)=\{0\}$. We have $D\left(X, 0_{2}\right)^{+}=\mathbb{R}^{2}, \partial g_{0}\left(0_{2}\right)=\operatorname{cl} B\left(0_{2}, 1\right)$, $A(\bar{x})=$ cone $\partial g_{0}\left(0_{2}\right)=\mathbb{R}^{2}=D\left(X, 0_{2}\right)^{-}$but (13) fails. So, LFMCQ holds at $\mathrm{O}_{2}$ while LSCQ fails.

Example 17 ([12, Example 25]) Consider the linear vector SIO problem

$$
\begin{array}{rll}
P: & \text { "min " } & f(x)=\left(x_{1}-x_{2},-x_{2}\right) \\
& \text { s.t. } & t x_{1}+\left(\sqrt{1-(t-1)^{2}}\right) x_{2} \leq 0, t \in[0,2],
\end{array}
$$

whose feasible set is $X=\mathbb{R}_{-}^{2}$. It follows that EKTCQ holds at $0_{2}$ (see [12, Example 25]) and $A\left(0_{2}\right)=\left(\mathbb{R}_{++} \times \mathbb{R}_{+}\right) \cup\left\{0_{2}\right\}$ is non-closed. Thus, EKTCQ does not imply $L F M C Q$. 
The following example shows that the converse statement of Theorem 15(iv) does not hold.

Example 18 Consider Example 12, for which $L S C Q$ is not fulfilled at $0_{2}$. We have

$$
\begin{aligned}
\varphi^{\prime}\left(0_{2} ; d\right) \leq 0 & \Leftrightarrow g_{t}^{\prime}\left(0_{2}, d\right) \leq 0 \forall t \in[0,1] \\
& \Leftrightarrow(t-1) d_{1}+d_{2} \leq 0 \text { and }(t-1) d_{1}-d_{2} \leq 0 \forall t \in[0,1] \\
& \Leftrightarrow d_{1} \geq 0, d_{2}=0 .
\end{aligned}
$$

Let $d \in \mathbb{R}^{2}$ be such that $\varphi^{\prime}\left(0_{2} ; d\right) \leq 0$. Then the vector function $h(s):=$ $\left(s d_{1}, s d_{2}\right)=\left(s d_{1}, 0\right) \in X$ for all $s \in\left[0,2 / d_{1}\right]$, satisfies $h(0)=0_{2}$ and $h^{\prime}(0)=d$. That is, $d \in A\left(X ; 0_{2}\right)$. So, EKTCQ holds at $0_{2}$.

Lemma 19 Let $\bar{x} \in \operatorname{bd} X$. Then, $P$ satisfies EKTCQ at $\bar{x}$ if and only if

$$
T(X ; \bar{x})=\left\{d \in \mathbb{R}^{n}: \varphi^{\prime}(\bar{x}, d) \leq 0\right\} .
$$

Proof: Assume that $P$ satisfies EKTCQ at $\bar{x}$, i.e.,

$$
\left\{d \in \mathbb{R}^{n}: \varphi^{\prime}(\bar{x}, d) \leq 0\right\} \subset A(X ; \bar{x})=T(X ; \bar{x}) .
$$

By (18) and Lemma 14, (17) holds. The converse statement is trivial.

The four constraint qualifications introduced above fail in the next example.

Example 20 Consider the following set

$$
X=\left\{x \in \mathbb{R}^{2}: g_{t}\left(x_{1}, x_{2}\right)=t x_{1}^{2}-t(1-t)+(1-t)\left(x_{2}^{2}+x_{2}\right) \leq 0, t \in[0,1]\right\} .
$$

Since

$$
g_{0}\left(x_{1}, x_{2}\right)=x_{2}^{2}+x_{2} \leq 0 \Rightarrow x_{2} \in[-1,0]
$$

and

$$
g_{1}\left(x_{1}, x_{2}\right)=x_{1}^{2} \leq 0 \Rightarrow x_{1}=0,
$$

one gets $X=\{0\} \times[-1,0]$. As $T\left(0_{2}\right)=\{0,1\}, 0_{2} \in \bigcup_{t \in T\left(0_{2}\right)} \partial g_{t}\left(0_{2}\right)=\{(0,0),(0,1)\}$

and so LSCQ fails at $0_{2}$. We also have $A\left(0_{2}\right)=$ cone $\{(0,1)\}$ and $D\left(X, 0_{2}\right)=$ cone $\{(0,-1)\}$, so that $A\left(0_{2}\right) \varsubsetneqq-D\left(X, 0_{2}\right)^{+}$, which implies the failure of LFMCQ. Since $g_{0}^{\prime}\left(0_{2} ; d\right)=d_{2}$ and $g_{1}^{\prime}\left(0_{2} ; d\right)=0,\left\{d \in \mathbb{R}^{2}: \varphi^{\prime}\left(0_{2} ; d\right) \leq 0\right\}=\mathbb{R} \times \mathbb{R}_{-}$. On the other hand $A\left(X ; 0_{2}\right)=\{0\} \times \mathbb{R}_{-}$. So, EKTCQ fails to hold at $0_{2}$.

\section{Cone conditions}

It is well-known (see, e.g. [9, Theorem 3.21 and Corollary 3.23]) that, if $T$ is finite, $f_{i}, i=1, \ldots, p$ and $g_{t}, t \in T$, are convex differentiable functions, $\bar{x} \in X$, and $0_{n} \notin \operatorname{conv}\left\{\nabla f_{1}(\bar{x}), \ldots, \nabla f_{p}(\bar{x})\right\}$, then $\bar{x}$ is a weakly efficient solution of the (ordinary) convex vector optimization problem $P$ if and only if there exist 
$t^{j} \in T(\bar{x}), j=1, \ldots, q$, as well as non-negative scalars $\lambda_{1}, \ldots, \lambda_{p}, \mu_{1}, \ldots, \mu_{q}$ satisfying

$$
\sum_{i=1}^{p} \lambda_{i} \nabla f_{i}(\bar{x})=\sum_{j=1}^{q} \mu_{j} \nabla g_{t^{j}}(\bar{x}) \neq 0_{n}
$$

In geometric terms, the KKT condition (19) asserts that $G(\bar{x}) \cap A(\bar{x}) \neq\left\{0_{n}\right\}$ (it is sufficient to take $q \leq n$ by Carathéodory's theorem applied to the convex cone $A(\bar{x}))$. In this section we give similar conditions for convex vector SIO problems and different types of efficiency.

The characterizations of efficient and weakly efficient solutions in this section extend similar results on linear vector SIO in [12] to convex vector SIO. We start with two sufficient conditions for $\bar{x} \in X$ to be efficient and weakly efficient solution independently of the constraints under assumptions which already appeared in Lemma 2.

Proposition 21 If $\bar{x} \in X$, then the following statements are true:

(i) If $0_{n} \in \operatorname{conv}\left(\bigcup_{i=1}^{p} \partial f_{i}(\bar{x})\right)$, then $\bar{x} \in X_{w E}$.

(ii) if $0_{n} \in \bigcup_{i=1}^{p} \partial f_{i}(\bar{x})$ and the components of $f$ are strictly convex, then $\bar{x} \in X_{E}$.

Proof: (i) Let $0_{n}=\sum_{i=1}^{p} \alpha_{i} \xi_{i}$, with $\alpha_{i} \geq 0$ and $\xi_{i} \in \partial f_{i}(\bar{x}), i=1, \ldots, p$, and $\sum_{i=1}^{p} \alpha_{i}=1$. Let $\alpha_{j}>0, j \in\{1, \ldots, p\}$. Then, $\xi_{j}=-\sum_{i \neq j} \frac{\alpha_{i}}{\alpha_{j}} \xi_{i}$. We can assume w.l.o.g. the existence of $i \neq j$ such that $\alpha_{i}>0$ (otherwise, $\xi_{j}=0_{n}$ and $\bar{x} \in X_{w E}$ because it is a minimizer of $f_{j}$ on $\mathbb{R}^{n}$, and so on $X$ ). If we suppose that there exists $\widehat{x} \in X$ such that $f(\widehat{x})<f(\bar{x})$, for every $i=1, \ldots, p$ and $\xi_{i} \in \partial f_{i}(\bar{x})$, then

$$
0>f_{i}(\widehat{x})-f_{i}(\bar{x}) \geq \xi_{i}^{\top}(\widehat{x}-\bar{x})
$$

Thus,

$$
0>\xi_{j}^{\top}(\widehat{x}-\bar{x})=-\sum_{i \neq j} \frac{\alpha_{i}}{\alpha_{j}} \xi_{i}^{\top}(\widehat{x}-\bar{x})>0
$$

which is a contradiction whereby $\bar{x} \in X_{w E}$.

(ii) If $0_{n} \in \bigcup_{i=1}^{p} \partial f_{i}(\bar{x})$ and all the objective functions are strictly convex, then $\bar{x}$ is the unique minimizer of at least one of the objective functions on $\mathbb{R}^{n}$ (and so on $X)$. Then, there is no $\widehat{x} \in X$ such that $f(\widehat{x}) \leq f(\bar{x})$, i.e., $\bar{x} \in X_{E}$.

Theorem 22 Let $\bar{x} \in X$ be such that $0_{n} \notin \bigcup_{i=1}^{p} \partial f_{i}(\bar{x})$. Then, the following statements hold:

(i) $\bar{x} \in X_{E}$ if and only if

$$
D(X ; \bar{x}) \cap G(\bar{x})^{-} \subset\left\{d \in \mathbb{R}^{n}: f_{i}^{\prime}(\bar{x} ; d)=0, i=1, \ldots, p\right\} .
$$


(ii) $\bar{x} \in X_{w E}$ if and only if $D(X ; \bar{x}) \cap \operatorname{int} G(\bar{x})^{-}=\emptyset$.

Proof: (i) Denote $F(\bar{x}):=\left\{d \in \mathbb{R}^{n}: f_{i}^{\prime}(\bar{x} ; d)=0, i=1, \ldots, p\right\}$.

Suppose that $\bar{x} \in X_{E}$. Let $d \in D(X ; \bar{x}) \cap G(\bar{x})^{-}$. Then, by Lemma 2(ii),

$$
f_{i}^{\prime}(\bar{x} ; d) \leq 0 \text {, for all } i=1, \ldots, p .
$$

Since $d \in D(X ; \bar{x})$ and $\bar{x} \in X_{E}$, if there exists $i_{0} \in\{1, \ldots, p\}$ such that $f_{i_{0}}^{\prime}(\bar{x} ; d)<0, d$ would be a feasible descent direction of $f_{i_{0}}^{\prime}$ at $\bar{x}$ and there would exist $j_{0} \in\{1, \ldots, p\}, j_{0} \neq i_{0}$, such that $d$ is a feasible ascent direction of $f_{j_{0}}$ at $\bar{x}$. Then, by Lemma $1, f_{j_{0}}^{\prime}(\bar{x} ; d)>0$, which contradicts (20). Thus $f_{i}^{\prime}(\bar{x} ; d)=0$, for all $i=1, \ldots, p$, i.e., $d \in F(\bar{x})$.

Now assume that $\bar{x} \notin X_{E}$. Then, there exist $d \in D(X ; \bar{x}), i_{0} \in\{1, \ldots, p\}$, and $\beta>0$ such that

$$
f_{i}(\bar{x}+\varepsilon d) \leq f_{i}(\bar{x}), i=1, \ldots, p,
$$

with

$$
f_{i_{0}}(\bar{x}+\varepsilon d)<f_{i_{0}}(\bar{x})
$$

for all $\varepsilon \in] 0, \beta\left[\right.$. From (21) it follows that $f_{i}^{\prime}(\bar{x} ; d) \leq 0, i=1, \ldots, p$. Thus, again by Lemma 2(ii), $d \in D(X ; \bar{x}) \cap G(\bar{x})^{-}$. But, from (22) we get $\varepsilon \xi_{i_{0}}^{\top} d \leq f_{i_{0}}(\bar{x}+$ $\varepsilon d)-f_{i_{0}}(\bar{x})<0$ for all $\xi_{i_{0}} \in \partial f_{i_{0}}(\bar{x})$. That is, $f_{i_{0}}^{\prime}(\bar{x} ; d)=\max _{\xi_{i_{0}} \in \partial f_{i_{0}}(\bar{x})} \xi_{i_{0}}^{\top} d<0$, so that $d \notin F(\bar{x})$.

(ii) If $0_{n} \in \operatorname{conv}\left(\bigcup_{i=1}^{p} \partial f_{i}(\bar{x})\right)$, then $\bar{x} \in X_{w E}$ by Proposition 21. So, we can assume that $0_{n} \notin \operatorname{conv}\left(\bigcup_{i=1}^{p} \partial f_{i}(\bar{x})\right)$, in which case $0_{n} \notin \bigcup_{i=1}^{p} \partial f_{i}(\bar{x})$. Let $d \in D(X ; \bar{x}) \cap$ int $G(\bar{x})^{-}$. Then, again by Lemma $2\left(\right.$ iii), $f_{i}^{\prime}(\bar{x} ; d)<0, i=1, \ldots, p$. That is, $d$ is a feasible descent direction for $f_{i}$ at $\bar{x}, i=1, \ldots, p$. So, $\bar{x} \notin X_{w E}$.

Now, suppose that $\bar{x} \notin X_{w E}$, i.e. there exists $x \in X$ such that $f_{i}(x)<f_{i}(\bar{x})$, $i=1, \ldots, p$. Let $d=x-\bar{x} \in D(X ; \bar{x})$. Since $d$ is a feasible descent direction for each $f_{i}$ at $\bar{x}$, it follows that $f_{i}^{\prime}(\bar{x} ; d)=\max _{\xi_{i} \in \partial f_{i}(\bar{x})} \xi_{i}^{\top} d<0$, again by Lemma 1. Thus, $d \in \operatorname{int} G(\bar{x})^{-}$by Lemma 2(iii). Thus, $D(X ; \bar{x}) \cap \operatorname{int} G(\bar{x})^{-} \neq \emptyset$, which completes the proof.

The following example shows that the assumption $0_{n} \notin \bigcup_{i=1}^{p} \partial f_{i}(\bar{x})$ in Theorem 22 is not superfluous.

Example 23 Let $n=2, p=2, f_{1}(x)=x_{1}^{2}+x_{2}^{2}, f_{2}(x)=x_{1}^{2}-2 x_{1}+x_{2}^{2}$, and $g_{t}(x)=x t_{1}^{2}-x_{2}+t-1$ for all $t \in[0,1]$. As $g_{t}$ grows with $t$,

$$
X=\left\{x \in \mathbb{R}^{2}: g_{1}(x) \leq 0\right\}=\left\{x \in \mathbb{R}^{2}: x_{2} \geq x_{1}^{2}\right\} .
$$


Since $f_{1}$ and $f_{2}$ are strictly convex and $0_{2}=\nabla f_{1}\left(0_{2}\right) \in \bigcup_{i=1,2} \partial f_{i}\left(0_{2}\right)$, we have $0_{2} \in X_{E}$. Now, take $d=(1,1)$. We have $D\left(X ; 0_{2}\right)=\mathbb{R} \times \mathbb{R}_{++} \cup\left\{0_{2}\right\}$ and $G\left(0_{2}\right)^{-}=\mathbb{R} \times \mathbb{R}_{+}$, so that $d \in D\left(X ; 0_{2}\right) \cap G\left(0_{2}\right)^{-}$, but $d \notin F\left(0_{2}\right)$ because $f_{2}^{\prime}\left(0_{2} ; d\right)=\nabla f_{2}\left(0_{2}\right)^{\top} d=-2 \neq 0$.

Corollary 24 Given $\bar{x} \in X, \bar{x} \in X_{w E}$ if and only if

$$
\left\{d \in \mathbb{R}^{n}: f_{i}^{\prime}(\bar{x} ; d)<0, i=1, \ldots, p\right\} \cap D(X ; \bar{x})=\emptyset .
$$

Proof: If $0_{n} \in \bigcup_{i=1}^{p} \partial f_{i}(\bar{x} ; d)$, then $\bar{x} \in X_{w E}$ by Proposition 21 and there exists $i \in\{1, \ldots, p\}$ such that $f_{i}^{\prime}(\bar{x} ; d) \geq 0$, so that (23) holds too. Otherwise, both statements are equivalent by Theorem 22(ii) and Lemma 2(iii).

For the sake of brevity, given $\lambda=\left(\lambda_{1}, \ldots, \lambda_{p}\right) \in \mathbb{R}^{p}$, we denote $\sum_{i=1}^{p} \lambda_{i} f_{i}$ in matrix form as $\lambda^{\top} f$.

Theorem 25 Given $\bar{x} \in X$, the following statements hold:

(i) $\bar{x} \in X_{p E}$ if and only if $\partial\left(\lambda^{\top} f\right)(\bar{x}) \cap D(X ; \bar{x})^{+} \neq \emptyset$ for some $\lambda>0_{p}$.

(ii) $\bar{x} \in X_{w E}$ if and only if $\partial\left(\lambda^{\top} f\right)(\bar{x}) \cap D(X ; \bar{x})^{+} \neq \emptyset$ for some $\lambda \geq 0_{p}$.

Proof: We associate with $P$ the parameterized (weighted) problem

$$
P(\lambda): \min \left(\lambda^{\top} f\right)(x)=\sum_{i=1}^{p} \lambda_{i} f_{i}(x) \text { s.t. } x \in X,
$$

where $\lambda \geq 0_{p}$ is the weight vector and $P(\lambda)$ is a convex SIO problem for each $\lambda$ (we could aggregate $\sum_{i=1}^{p} \lambda_{i}=1$ ). By Theorem 27.4 of [27] it follows that $\bar{x}$ is an optimal solution of $P(\lambda)$ for some $\lambda \geq 0_{p}$ if and only if there exists $\xi \in \partial\left(\lambda^{\top} f\right)(\bar{x}) \cap D(X ; \bar{x})^{+}$.

(i) According to the Geoffrion Theorem ([11], see also [9, Theorem 3.15]), $\bar{x}$ is an optimal solution of $P(\lambda)$ for some $\lambda>0_{p}$ if and only if $\bar{x} \in X_{p E}$.

(ii) Similarly, by [9, Proposition 3.10], $\bar{x}$ is an optimal solution of $P(\lambda)$ for some $\lambda \geq 0_{p}$ if and only if $\bar{x} \in X_{w E}$.

Observe that, given $\bar{x} \in X, 0_{n} \in \operatorname{conv}\left(\bigcup_{i=1}^{p} \partial f_{i}(\bar{x})\right)$ entails that $X_{w E}$. We consider now the case where $0_{n} \notin \operatorname{conv}\left(\bigcup_{i=1}^{p} \partial f_{i}(\bar{x})\right)$.

Corollary 26 Let $\bar{x} \in X$ be such that $0_{n} \notin \operatorname{conv}\left(\bigcup_{i=1}^{p} \partial f_{i}(\bar{x})\right)$. Then $\bar{x} \in X_{w E}$ if and only if $G(\bar{x}) \cap D(X, \bar{x})^{+} \neq\left\{0_{n}\right\}$, in which case $\bar{x} \in$ bd $X$. 
Proof: Let $0_{n} \notin \operatorname{conv}\left(\bigcup_{i=1}^{p} \partial f_{i}(\bar{x})\right)$. Then, $G(\bar{x}) \cap D(X, \bar{x})^{+} \neq\left\{0_{n}\right\}$ if and only if there exist $\lambda \geq 0_{p}, \xi_{i} \in \partial f_{i}(\bar{x}), i=1, \ldots, p$ such that $\sum_{i=1}^{p} \lambda_{i} \xi_{i} \in D(X ; \bar{x})^{+}$if and only if

$$
\sum_{i=1}^{p} \lambda_{i} \partial f_{i}(\bar{x}) \cap D(X ; \bar{x})^{+}=\partial\left(\lambda^{\top} f(\bar{x})\right) \cap D(X ; \bar{x})^{+} \neq\left\{0_{n}\right\}
$$

for some $\lambda \geq 0_{p}$. The first statement follows from Theorem 25(ii).

Finally, if $\bar{x} \in \operatorname{int} X$, then $D(X ; \bar{x})^{+}=\left\{0_{n}\right\}$, so that $G(\bar{x}) \cap D(X, \bar{x})^{+}=$ $\left\{0_{n}\right\}$. Hence $\bar{x} \notin X_{w E}$ by the first statement.

\section{$5 \quad$ KKT conditions}

We are in a position to obtain KKT optimality conditions.

Theorem 27 Given $\bar{x} \in X$, the following statements hold:

(i) If there exists $\lambda \geq 0_{p}\left(\lambda>0_{p}\right)$ satisfying

$$
(K K T) \quad \partial\left(\lambda^{\top} f\right)(\bar{x}) \cap(-A(\bar{x})) \neq \emptyset,
$$

then $\bar{x} \in X_{w E}\left(\bar{x} \in X_{p E}\right.$, respectively).

(ii) If $\bar{x} \in X_{w E}\left(\bar{x} \in X_{p E}\right)$ satisfies LFMCQ, then there exist $\lambda \geq 0_{p}\left(\lambda>0_{p}\right.$, respectively) such that (KKT) holds. If, additionally, $0_{n} \notin \operatorname{conv}\left(\bigcup_{i=1}^{p} \partial f_{i}(\bar{x})\right)$, then the following stronger condition holds:

$$
\partial\left(\lambda^{\top} f\right)(\bar{x}) \cap(-A(\bar{x})) \neq\left\{0_{n}\right\} .
$$

Proof: (i) Recall that $D(X ; \bar{x}) \subset A(\bar{x})^{-}$. Taking positive polars we get

$$
D(X ; \bar{x})^{+} \supset-A(\bar{x})^{++}=-\operatorname{cl} A(\bar{x}) \supset-A(\bar{x}),
$$

so that $(\mathrm{KKT})$ implies that $\partial\left(\lambda^{\top} f\right)(\bar{x}) \cap D(X ; \bar{x})^{+} \neq \emptyset$. The conclusion follows from Theorem 25.

(ii) We are assuming that $A(\bar{x})=D(X ; \bar{x})^{-}=-D(X ; \bar{x})^{+}$. The first part is straightforward consequence of Theorem 25 while the second one follows from the argument of Corollary 26.

Lemma 28 If $\bar{x} \in X_{w E}$, then

$$
\left\{d \in \mathbb{R}^{n}: f_{i}^{\prime}(\bar{x} ; d)<0, i=1, \ldots, p\right\} \cap T(X ; \bar{x})=\emptyset .
$$


Proof: Assume the contrary, that is, there exists $\bar{d} \in T(X ; \bar{x})$ satisfying

$$
f_{i}^{\prime}(\bar{x} ; \bar{d})<0, i=1, \ldots, p .
$$

By $\bar{d} \in T(X ; \bar{x})$, there exist sequences $\left\{s^{k}\right\}_{k \in \mathbb{N}}$ and $\left\{d^{k}\right\}_{k \in \mathbb{N}}$ such that $s^{k} \downarrow 0$, $d^{k} \rightarrow \bar{d}$ and $\bar{x}+s^{k} d^{k} \in X$ for all $k \in \mathbb{N}$. Since $\bar{x} \in X_{w E}$ there exists (perhaps after passing to a subsequence) an index $i_{0} \in\{1, \ldots, p\}$ such that

$$
f_{i_{0}}\left(\bar{x}+s^{k} d^{k}\right) \geq f_{i_{0}}(\bar{x}), k \in \mathbb{N} .
$$

Since $f_{i_{0}}$ is directional differentiable at $\bar{x}$ in the Hadamard sense (see e.g. [1, Proposition 2.126(v)(c)]), the latter inequalities provide

$$
f_{i_{0}}^{\prime}(\bar{x} ; \bar{d})=\lim _{k \rightarrow \infty} \frac{f_{i_{0}}\left(\bar{x}+s^{k} d^{k}\right)-f_{i_{0}}(\bar{x})}{s^{k}} \geq 0
$$

which contradicts (26).

Observe that, since $D(X ; \bar{x}) \subset T(X ; \bar{x})$, the direct part of Corollary 24 is immediate consequence of Lemma 28.

We have shown in Theorem 27 that (KKT) is a necessary condition for weak efficiency under LFMCQ (and, by Lemma 8, also under SCQ). Finally, we prove that this necessary condition still holds under the remaining two CQ introduced in Section 3, namely, LSCQ and EKTCQ, together with the closedness of the active cone recall that LSCQ entails the latter property according to Corollary $11)$.

Theorem 29 Let $0_{n} \notin \operatorname{conv}\left(\bigcup_{i=1}^{p} \partial f_{i}(\bar{x})\right)$ and $\bar{x} \in X_{w E}$ satisfying one of the following conditions:

(i) $L S C Q$;

(ii) EKTCQ and $A(\bar{x})$ is closed.

Then, there exists $\lambda \geq 0_{p}$ satisfying (KKT).

Proof: Since $\bar{x} \in X_{w E}$ and $0_{n} \notin \operatorname{conv}\left(\bigcup_{i=1}^{p} \partial f_{i}(\bar{x})\right), \bar{x} \in \operatorname{bd} X$ and

$$
\left\{d \in \mathbb{R}^{n}: \sum_{i=1}^{p} \lambda_{i} f_{i}^{\prime}(\bar{x} ; d)<0, \text { for all } \lambda \geq 0_{p}\right\} \cap T(X ; \bar{x})=\emptyset
$$

by Corollary 26 and Lemma 28, respectively. Combining the formulas (15) and (27) with Theorem 15(iv) and Lemma 19, we conclude that there is no $d \in \mathbb{R}^{n}$ such that

$$
\sum_{i=1}^{p} \lambda_{i} f_{i}^{\prime}(\bar{x} ; d)<0 \text { for all } \lambda \geq 0_{p}
$$

and

$$
g_{t}^{\prime}(\bar{x} ; d) \leq 0 \text { for all } t \in T(\bar{x}) .
$$

(i) Assume that LSCQ is satisfied at $\bar{x}$. Now, (28)-(29) is equivalent to: there is no $d \in \mathbb{R}^{n}$ such that 


$$
\xi^{\top} d<0 \text { for all } \xi \in \partial\left(\lambda^{\top} f\right)(\bar{x}) \text { for all } \lambda \geq 0_{p}
$$

and

$$
\xi^{\top} d \leq 0 \text { for all } \xi \in \partial g_{t}(\bar{x}) \text { for all } t \in T(\bar{x})
$$

Since the homogeneous linear system formed by (31)-(30) is inconsistent, $\partial\left(\lambda^{\top} f\right)(\bar{x})$ is a compact convex set and $A(\bar{x}):=$ cone $\left(\bigcup_{t \in T(\bar{x})} \partial g_{t}(\bar{x})\right)$ is closed (by Corollary 11), so that the Minkowski sum of both sets is closed, we can apply Motzkin's Theorem [13, Theorem 3.5] to conclude that

$$
0_{n} \in \partial\left(\lambda^{\top} f\right)(\bar{x})+A(\bar{x})
$$

(ii) The proof is the same, taking into account that now $A(\bar{x})$ is closed by assumption.

Example 17 shows that the closedness assumption in Theorem 29(ii) is not superfluous. Indeed, the unique solution of the system formed by the non-linear equations

$$
\lambda_{1}\left(\begin{array}{c}
1 \\
-1
\end{array}\right)+\lambda_{2}\left(\begin{array}{c}
0 \\
-1
\end{array}\right)=\sum_{i=1}^{2} \mu_{i}\left(\begin{array}{c}
-t^{i} \\
-\sqrt{1-\left(t^{i}-1\right)^{2}}
\end{array}\right), t^{1}, t^{2} \in[0,2],
$$

and the inequalities

$$
\lambda_{i} \geq 0, \mu_{i} \geq 0, i=1,2,
$$

is $\lambda_{1}=\lambda_{2}=\mu_{1}=\mu_{2}=0$. Thus, (KKT) fails.

Example 30 Consider the robust counterpart problem in (2) with $\mathcal{U}_{i}=B\left(\bar{c}_{i}, \varepsilon\right) \subset$ $\mathbb{R}^{n}, i=1, \ldots, p$. Then, $f_{i}(x)=\max _{c_{i} \in \mathcal{U}_{i}} c_{i}^{\top} x=\bar{c}_{i}^{\top} x+\varepsilon\|x\|$, with

$$
\partial f_{i}(x)= \begin{cases}\left\{\bar{c}_{i}+\varepsilon \frac{x}{\|x\|}\right\}, & \text { if } x \neq 0_{n} \\ B\left(\bar{c}_{i}, \varepsilon\right), & \text { if } x=0_{n} .\end{cases}
$$

Let $X=\left\{x \in \mathbb{R}^{n}: p_{k}^{\top} x \geq q_{k}, k \in K\right\}$ be the feasible set of (2) and let $\bar{x} \in$ $X$. According to Theorem 15, Proposition 21, and Theorems 27 and 29, the following statements hold:

(i) If $0_{n} \in \operatorname{conv}\left(\bigcup_{i=1}^{p} \partial f_{i}(\bar{x})\right)$, then $\bar{x}$ is a minmax robust weakly efficient solution.

(ii) Assume that $0_{n} \notin \operatorname{conv}\left(\bigcup_{i=1}^{p} \partial f_{i}(\bar{x})\right)$ and either LSCQ or EKTCQ holds at $\bar{x} \neq 0_{n}$. Then, $\bar{x}$ is a weakly efficient solution of (2) if and only if there exists $\lambda \geq 0$ such that $-\sum_{i=1}^{p} \lambda_{i}\left(\bar{c}_{i}+\varepsilon \frac{\bar{x}}{\|\bar{x}\|}\right) \in A(\bar{x})$. 


\section{References}

[1] J.F. Bonnans, A. Shapiro, Perturbation analysis of optimization problems, Springer, Berlin, 2000.

[2] R.I. Boţ, S.-M. Grad, G. Wanka, Duality in vector optimization, Springer, Berlin, 2009.

[3] G. Caristi, M. Ferrara, A. Stefanescu, Semi-infinite multiobjective programming with generalized invexity, Math Reports 12 (2010) 217-233.

[4] Chuong, T.D., Huy, N.Q., \& Yao, J.C. (2009). Stability of semi-infinite vector optimization problems under functional perturbations. J. Glob. Optim., 45, 583-595.

[5] T.D. Chuong, N.Q. Huy, J.C. Yao, Pseudo-Lipschitz property of linear semi-infinite vector optimization problems, Eur J Oper Res 200 (2010) 639-644.

[6] T.D. Chuong, N.Q. Huy, J.C. Yao, (2010). Stability of semi-infinite vector optimization problems under functional perturbations. J. Global Optim, $45,583-595$.

[7] T.D. Chuong, D.S. Kim, Nonsmooth semi-infinite multiobjective optimization problems, J Optim Theory Appl 160 (2014) 748-762.

[8] N. Dinh, M.A. Goberna, M.A. López, T.Q. Son, New Farkas-type constraint qualifications in convex infinite programming, ESAIM Control Optim Calc Var 13 (2007) 580-597.

[9] M. Ehrgott, Multicriteria optimization (2nd ed.), Springer, Berlin, 2005.

[10] X. Fan, C. Cheng, H. Wang, Density of stable semi-infinite vector optimization problems under functional perturbations, J Global Optim, to appear.

[11] A. Geoffrion, Proper efficiency and the theory of vector maximization, J Math Anal Appl 22 (1968) 618-630.

[12] M.A. Goberna, F. Guerra-Vazquez, M.I. Todorov, Constraint qualifications in linear vector semi-infinite optimization, Eur J Oper Res 227 (2013) 12-21.

[13] M.A. Goberna, M.A. López, Linear semi-infinite optimization, Chichester: Wiley, 1998.

[14] F. Guerra-Vazquez, J.-J. Rückmann, On proper efficiency in multiobjective semi-infinite optimization. in: H. Xu, K.L. Teo, Y. Zhang, (Eds.), Optimization and control techniques and applications, Springer, Berlin, 2014, pp. 115-135.

[15] M. Ehrgott, J. Idec, A. Schöbel, Minmax robustness for multi-objective optimization problems, Eur J Oper Res 239 (2014) 17-31. 
[16] A. Hantoute, M.A. López, A complete characterization of the subdifferential set of the supremum of an arbitrary family of convex functions, J. Convex Anal 15 (2008) 831-858.

[17] R. Hartley, On cone-efficiency, cone-convexity and cone-compactness, SIAM J Appl Math 34 (1978) 211-222.

[18] J.B. Hiriart-Urruty, C . Lemarechal, Convex analysis and minimization algorithms I. Springer, N.Y., 1993.

[19] H. Kuhn, A. Tucker, Nonlinear programming, in: J. Newman (Ed.), Proceedings of the second Berkeley symposium on mathematical statistics and probability, Berkeley: University of California Press, 1951, pp. 481-492.

[20] P.J. Laurent, Approximation et optimisation (French), Hermann, Paris, 1972.

[21] C. Li, K.F. Ng, On constraint qualification for an infinite system of convex inequalities in a Banach space, SIAM J Optim 15 (2005) 488-512.

[22] C. Li, K.F. Ng, T.K. Pong, Constraint qualifications for convex inequality systems with applications in constrained optimization, SIAM J Optim 19 (2008) 163-187.

[23] C. Li, X. Zhao, Y. Hu, Quasi-Slater and Farkas-Minkowski qualifications for semi-infinite programming with applications, SIAM J Optim 23 (2013) $2208-2230$.

[24] J.E. Martínez-Legaz, M.I. Todorov, C.A. Zetina, $\gamma$-Active constraints in convex semi-infinite programming, Numer. Funct.l Anal. Optim. 35 (2014) 1078-1094.

[25] D.W. Peterson, A review of constraint qualifications in finite-dimensional spaces, SIAM Review 15 (1973) 639-654.

[26] R. Puente, V.N. Vera de Serio, Locally Farkas-Minkowski linear inequality systems, Top 7 (1999) 103-121.

[27] R.T. Rockafellar, Convex analysis, Princeton U.P., Princeton, 1970.

[28] O. Stein, First-order optimality conditions for degenerate index sets in generalized semi-infinite optimization. Math. Oper. Res., 26 (2001) 565582.

[29] R.A. Tapia, M.W. Trosset, An extension of the Karush-Kuhn-Tucker necessity conditions to infinite programming, SIAM Review 36 (1994) 1-17.

[30] M.I. Todorov, Well-posedness in the linear vector semi-infinite optimization, in G.H. Tzeng (Ed.), Multiple criteria decision making, Springer, N.Y., pp. 141-150, 1994. 
[31] M.I. Todorov, Kuratowksi convergence of the efficient sets in the parametric linear vector semi-infinite optimization, Eur J Oper Res 94 (1996). 610-617.

[32] C. Zălinescu, Convex analysis in general vector spaces. World Scientific Publishing Co., New Jersey, 2002. 